\title{
Expecting confirmed and death cases of covid-19 in Iraq by utilizing backpropagation neural network
}

\author{
Moatasem Yaseen Al-Ridha ${ }^{1}$, Ammar Sameer Anaz ${ }^{2}$, Raid Rafi Omar Al-Nima ${ }^{3}$ \\ ${ }^{1,3}$ Technical Engineering College, Northern Technical University, Mosul, Iraq \\ ${ }^{2}$ Basic Education College, University of Mosul, Mosul, Iraq
}

\section{Article Info \\ Article history: \\ Received Feb 16, 2021 \\ Revised Apr 13, 2021 \\ Accepted May 27, 2021}

Keywords:

Backpropagation neural network

Covid-19

Prediction

\begin{abstract}
The world is currently facing a strong epidemic and pandemic of coronavirus. This motivates establishing our paper, where this virus pushes researchers to study, investigate, observe, analyse and try solving its related issues. In this work, an artificial neural network (ANN) model of backpropagation neural network (BNN) with two hidden layers is proposed for expecting confirmed cases and death cases of coronavirus disease 2019 (covid-19). As a field of study, Iraq country has been considered in this paper. Covid-19 dataset from our world in data (OWID) is used here. Promising result is achieved where a very small error value of 0.0035 is reported in overall the evaluations. This paper may implicate establishing further researches that consider other parameters and other countries over the world. It is worth mentioning that the suggested ANN model may help decision maker people in taking quarantine movements against the strong epidemic and pandemic of covid- 19 .
\end{abstract}

This is an open access article under the CC BY-SA license.

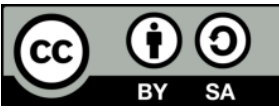

Corresponding Author:

Moatasem Yaseen Al-Ridha

Technical Engineering College

Northern Technical University, Mosul, Iraq

Email: Moatasem@ntu.edu.iq

\section{INTRODUCTION}

The world is currently challenging an epidemic of an unusual virus called covid-19. Organizations have become more pressing than ever as humanity need a streamline patient analysis. In literature, prediction of covid-19 was studied in several papers as in [1]-[5]. Ganavi et al. proposed a global systematic literature review to summarize trends in the modeling techniques used for covid-19 from the 1st of January 2020 to the 30th of June 2020. The authors examined many models of predictions such as confidence interval (CI) or credibility interval (CrI), the reliability and correctness of comparing values should be used with cautious [6]. M. Castro, S. Ares, J. A. Cuesta, and S. Manrubia suggested a study that advises against accurate forecasts of the evolution of epidemics that found in phenomenological models, mean-field or effective. Only probabilities of different outcomes can confidently be given [7]. Matta and Saraf offered a set of recognizing algorithms such as support vector machines (SVM), random forests (RF), artificial neural network (ANN) for prediction. RF got better performance among them [8]. W. C. Roda, M. B. Varughese, D. Han, and M. Y. Li proposed that the key reason for wide differences in predictions model caused by the non-identifiability in model calibrations using the confirmed-case data [9]. Calistus N. Ngonghala, Enahoro Iboi, Steffen Eikenberry, et al., discussed the Non-pharmaceutical interventions strategies to control and reduce the effect of the mitigating the burden of the pandemic. The mathematical model depends on the deterministic system of nonlinear differential equations for assessing the population-level impact of controlling [10]. S. Boccaletti, W. Ditto, G. Mindlin, and A. Atangana presented a study of forecasting and modeling epidemics. Pnademics are considered here as covid-19 [11]. P. Melin, J. C. Monica, D. Sanchez, and O. Castillo suggested a model 
of multiple ensemble neural network with fuzzy response aggregation for the covid-19. Ensemble neural networks were composed of a set of modules, which are used to produce several predictions under different conditions to improve the final prediction by combining the outputs of the modules in an intelligent way. Fuzzy logic handled the uncertainty in the process of making the final prediction decision [12]. Sun and Wang offered a model that matched the epidemic data in heilongjiang province. It was found that continuously strict measures indeed impede the covid-19 spreading [13]. Castillo and Melin adopted a hybridintelligent approach, which is composed of the fractal dimension definition and fuzzy logic concepts for achieving a classification accurate for countries based on the complexity of covid-19 time series data. Moreover, the results show that a classification accuracy could be further enhanced by the hybridintelligent approach [14]. Castillo and Melin described a hybrid intelligent method for forecasting covid-19 time series by combining fractal theory and fuzzy logic. Provided results can help decision makers to deal with the pandemic [15].

The aim, contribution and originality of this work is presenting an intelligent neural network of BNN to expect the confirmed and death cases of a serious pandemic (the covid-19) in one of middle east countries (Iraq). This can be performed by providing inputs of dates (as employed months and days) and outputs of expected cases (as numbers of confirmed and death cases). The remaining sections after the introduction are distributed as follows: section 2 explains the employed BNN, section 3 discusses the results and section 4 yields the conclusion of this paper.

\section{PROPOSED BNN}

In this paper, the general architecture of the proposed BNN for confirmed and death cases of covid-19 in Iraq is given in Figure 1.

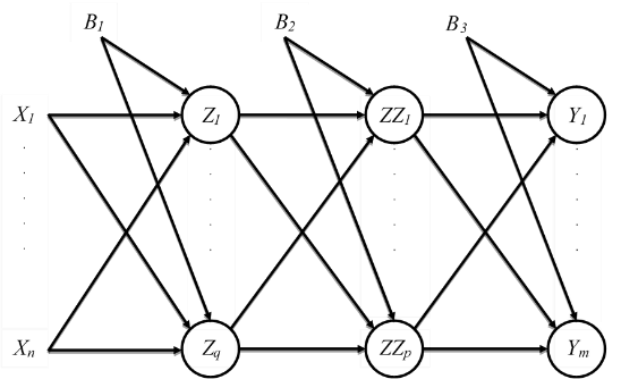

Figure 1. The general architecture of the proposed BNN

\subsection{Training algorithm}

The training algorithm of the BNN that consists of two hidden layers can be described as [16]. Feedforward stage:

$$
\begin{aligned}
& Z_{-} i n_{h}=u_{0 h}+\sum_{i=1}^{n} X_{i} u_{i h} \\
& Z_{h}=f\left(z_{-} i n_{h}\right) \\
& Z_{-} i n_{j}=v_{0 j}+\sum_{h=1}^{q} Z_{h} v_{h j} \\
& Z Z_{j}=f\left(z z_{-} i n_{j}\right) \\
& y_{-} i n_{k}=w_{0 k}+\sum_{j=1}^{p} Z Z_{j} w_{j k} \\
& Y_{k}=f\left(y_{-} i n_{k}\right)
\end{aligned}
$$

where $z_{-} i n_{h}$ is the inputs of the first hidden layer $(h=1, \ldots, q), u_{0 h}$ is the connection weights between the first bias $B_{l}$ and first hidden layer, $X_{\mathrm{i}}$ is the inputs $(i=1, \ldots, n), u_{i h}$ is the connection weights between the input and first hidden layer, $Z_{h}$ is the transfer function outputs of the first hidden layer, $z z_{-} i n_{\mathrm{j}}$ is the inputs of the second hidden layer $(h=1, \ldots, q), v_{0 j}$ is the connection weights between the second bias $B_{2}$ and second hidden layer, $v_{h j}$ 
is the connection weights between the first hidden and second hidden layers, $Z Z_{j}$ is the transfer function outputs of the second hidden layer, $y_{-} i n_{k}$ is the inputs of the output layer $(k=1, \ldots, m)$, $w_{0 k}$ is the connection weights between the third bias $B_{3}$ and output layer, $w_{j k}$ is the connection weights between the second hidden and output layers, and $Y_{k}$ is the transfer function of the output layer. All the weights here are initialized as small random numbers. Backpropagation of error stage:

$$
\begin{aligned}
& \delta_{k}=\left(T_{k}-Y_{k}\right) f^{\prime}\left(y_{-} i n_{k}\right) \\
& \Delta w_{j k}=\alpha \delta_{k} Z Z_{j} \\
& \Delta w_{0 k}=\alpha \delta_{k} \\
& \delta_{-} i n_{j}=\sum_{k=1}^{m} \delta_{k} w_{j k} \\
& \delta_{j}=\delta_{-} i n_{j} f^{\prime}\left(z z_{-} i n_{j}\right) \\
& \Delta v_{n j}=\alpha \delta_{j} Z_{n} \\
& \Delta v_{0 j}=\alpha \delta_{j} \\
& \delta_{-} i n_{h}=\sum_{j=1}^{p} \delta_{j} v_{h j} \\
& \delta_{h}=\delta_{-} i n_{h} f^{\prime}\left(z_{-} i n_{h}\right) \\
& \Delta u_{i h}=\alpha \delta_{h} X_{i} \\
& \Delta v_{0 j}=\alpha \delta_{j}
\end{aligned}
$$

where $\delta_{k}$ is the output error in the output layer, $T_{k}$ is the provided targets, $f^{\prime}\left(y_{-} i n_{k}\right)$ is the derivative function of $f\left(y_{-} i n_{k}\right), \Delta$ is the updating computations, $\alpha$ is the learning rate, $\delta_{-} i n_{j}$ is the input error in the second hidden layer, $\delta_{j}$ is the second error in the second hidden layer, $f^{\prime}\left(z z_{-} i n_{j}\right)$ is the derivative function of $f\left(z z_{-} i n_{j}\right), \delta_{-} i n_{h}$ is the input error in the first hidden layer, $\delta_{h}$ is the first error in the first hidden layer and $f^{\prime}\left(z_{-} i n_{h}\right)$ is the derivative function of $f\left(z_{-} i n_{h}\right)$. Update biases and weights stage:

$$
\begin{aligned}
& w_{j k}(\text { new })=w_{j k}(\text { old })+\Delta w_{j k} \\
& w_{0 k}(\text { new })=w_{0 k}(\text { old })+\Delta w_{0 k} \\
& v_{h j}(\text { new })=v_{h j}(\text { old })+\Delta v_{h j} \\
& v_{0 j}(\text { new })=v_{0 j}(\text { old })+\Delta v_{0 j} \\
& u_{i h}(\text { new })=u_{i h}(\text { old })+\Delta u_{i h} \\
& u_{0 h}(\text { new })=u_{0 h}(\text { old })+\Delta u_{0 h}
\end{aligned}
$$

\subsection{Testing algorithm}

The testing algorithm of the BNN that consists of two hidden layers can be illustrated as [16].

$$
\begin{aligned}
& z_{-} i n_{h}=u_{0 h}+\sum_{i=1}^{n} X_{i} u_{i h} \\
& Z_{h}=f\left(z_{-} i n_{h}\right) \\
& z z_{-} i n_{j}=v_{0 j}+\sum_{h=1}^{q} Z_{h} v_{h j} \\
& Z Z_{j}=f\left(z z_{-} i n_{j}\right)
\end{aligned}
$$




$$
\begin{aligned}
& y_{-} i n_{k}=w_{0 k}+\sum_{j=1}^{p} Z Z_{j} w_{j k} \\
& Y_{k}=f\left(y_{-} i n_{k}\right)
\end{aligned}
$$

where the weights here are initialized by the obtained values from the training algorithm.

\subsection{Proposed BNN specifications}

The specifications of the proposed BNN are as follows: the inputs are for the date of month $\left(X_{1}\right)$ and day $\left(X_{2}\right)$, the outputs are for the expected confirmed cases $\left(Y_{1}\right)$ and death cases $\left(Y_{2}\right)$, two hidden layers $(q=p=2)$, the activation function for the first and second hidden layers is bipolar sigmoid, the activation function for the output layer is pure linear, and the testing error type between the outputs and targets is the mean squared error (MSE). It is worth mentioning that in order to avoid the over loading in the BNN, the inputs and outputs are normalized by reducing their values.

\section{RESULTS AND DISCUSSIONS}

First of all, this type of epidemic does require a comprehensive dataset in order to establish a useful study. In this paper, the used covid-19 dataset is taken from the our world in data (OWID) [17]. The original dataset has various records from all over the world such as information for date, total cases, new cases, new cases smoothed, total deaths, new deaths, new deaths smoothed, total cases per million, new cases per million, new cases smoothed per million, total deaths per million, new deaths per million, new deaths smoothed per million, new tests, total tests, total tests per thousand, new tests per thousand, new tests smoothed, new tests smoothed per thousand, tests per case and positive rate.

In this work, we have focused on the information that belong to the country of Iraq for the period of $1^{\text {st }}$ January 2020 to $13^{\text {th }}$ October 2020 . As mentioned, only the data of months, days, new or confirmed cases, and new or confirmed deaths. BNN training data has been selected from the odd orders and testing data has been determined from the even orders.

Figure 2 demonstrates the relationship between the targets and outputs. It can be seen from this figure that the attained outputs are so close from the targets. Obviously, the regression (R) factor is here equal to approximately 0.95 and this is a further evidence about how the training is succeeded as the optimal $\mathrm{R}$ value is equal to 1 . It can be observed from Figure 3 that the training curve is successfully reduced from a big error value to a very small error value of 0.0035 in 13 epochs. This curve represents the relationship between the number of epochs and MSE. It can be considered that the training is succeeded in reaching the desired goal.

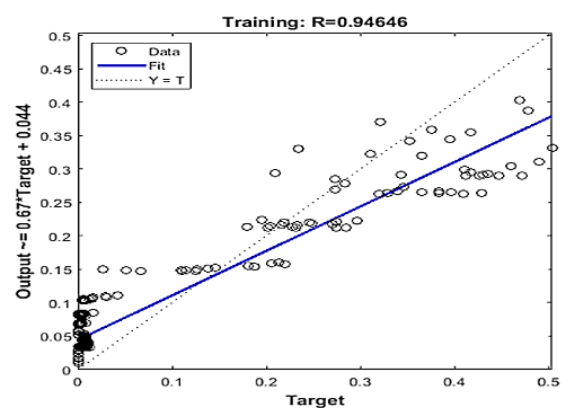

Figure 2. Regression outcomes between the targets and outputs of the suggested BNN

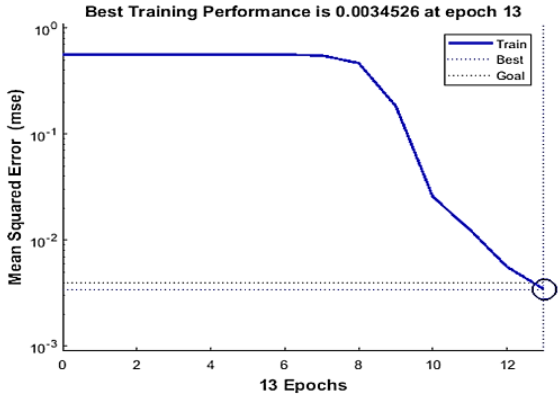

Figure 3. Training progress for the proposed BNN

Figure 4 depicts three shapes for the BNN model along the training epochs. Firstly, the gradient curve which is for the computed direction and magnitude that is utilized to adjust the weights by the right amount and in the right direction as explained in [16]. It is reasonably decreased to a very small error value of 0.05 . Secondly, the mu curve which is a training algorithm control parameter as illustrated in [18]. It is also successfully reduced to a very small value of 0.00005 .

In the BNN evaluations, such interesting and promising results were achieved. That is, for expecting the covid-19 values of confirmed cases and death cases in Iraq, a very small error of 0.0035 is benchmarked 
to all the evaluations. The proposed model can help responsible people of taking actions against the covid-19 as in providing logistical supplies and necessary medical quarantines. A stacked lines chart of testing BNN outcomes is shown in Figure 5.

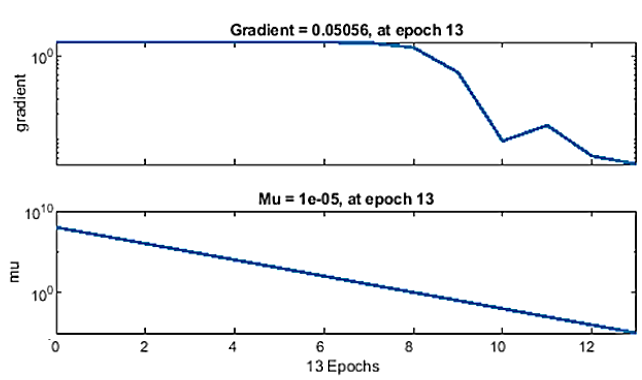

Figure 4. Progressing of training parameters for the proposed BNN

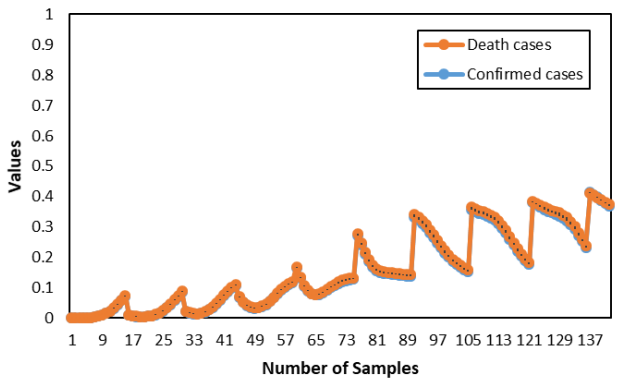

Figure 5. Stacked lines chart of testing BNN outcomes

This figure depicts a stacked lines chart of testing BNN outcomes. It demonstrates testing rhythm of BNN outputs. It yields how employed covid-19 cases are fluctuated and gradually increased. Comparison between different outcomes of ANNs is given in Table 1.

Table 1. Comparison between different outcomes of ANNs

\begin{tabular}{lccc}
\hline \multicolumn{1}{c}{ Neural Network } & Training error & Epochs & Testing error \\
\hline Cascade-forward & 0.0040 & 14 & 0.0040 \\
Bayesian Regularization & 0.0041 & 44 & 0.0039 \\
Radial Basis Functions & 0.0040 & 49 & 0.0039 \\
Our proposed model & 0.0035 & 13 & 0.0035 \\
\hline
\end{tabular}

From this table it can be seen that reasonable results are reported. Cascade-forward attained high training and testing errors of 0.0040 . This can be due to the architecture of such neural network type, which broadcasts weights from each layer (input and hidden(s)) to the output layer. This type of neural network does not require many training epochs to reach its goals. ANN of bayesian regularization also obtained high training and testing errors of 0.0041 and 0.0039 , respectively. The reason behind this can be illustrated by the role of such ANN, which concentrates on smoothing the outputs and this may lead to unprescise outputs. A long training time was spent as the number of epochs here equal to 44 and this is because of its unfast training algorithm. ANN of radial basis functions also achieved high training and testing errors of 0.0040 and 0.0039 , respectively. This can be explained by the nature of such ANN as it is sensitive to the training targets. This compared network also spent long training time where the number of epochs here was reached to 49. Our proposed BNN has recorded best performances according to other compared neural networks. It has attaind a small training and testing error of 0.0035 . Furthermore, it spent very short training time where it achieved 13 epochs only to reach its goals. It is worth mentioning that the proposed BNN model can be counted as one of efficient models in [19]-[35].

\section{CONCLUSION}

A strategy to predict the confirmed and death cases of a severe pandemic (covid-19) in one of Middle-East countries (Iraq) was proposed. In this paper, an intelligent neural network called the BNN was employed. One of the most significant findings in this study is that the suggested BNN could produce expected outcomes of confirmed and death cases with a very small error value (0.0035). It can be yield that the suggested model may support decision makers to take necessary quarantine actions against the epidemic of covid-19. The new version of covid-19, or as it called covid-20, is a thing that can directly be considered in future work after receiving sufficient recorded database. Moreover, additional predicting studies may be established for total cases, new cases smoothed, total deaths, new deaths smoothed, total cases per million, new cases smoothed per million, total deaths per million, new deaths smoothed per million, total tests, total tests per thousand, tests per case or positive rate. 


\section{ACKNOWLEDGMENTS}

Technical Engineering College/Northern Technical University/Mosul/Iraq, Basic Education College/University of Mosul/Iraq and Mr. H. Ritchie with the OWID for the available dataset of Coronavirus.

\section{REFERENCES}

[1] Laure Wynants, Ben Van Calster, Gary S Collins, Richard D Riley, Georg Heinze, Ewoud Schuit, et al., "Prediction models for diagnosis and prognosis of covid-19: systematic review and critical appraisal," 2020, doi: 10.1136/bmj.m1328.

[2] P. Arora, H. Kumar, and B. K. Panigrahi, "Prediction and analysis of COVID-19 positive cases using deep learning models: A descriptive case study of India," Chaos, Solitons and Fractals, vol. 139, 2020, doi: 10.1016/j.chaos.2020.110017.

[3] X. Zhang, R. Ma, and L. Wang, "Predicting turning point, duration and attack rate of COVID-19 outbreaks in major Western countries," Chaos, Solitons and Fractals, vol. 135, 2020, doi: 10.1016/j.chaos.2020.109829.

[4] B. F. Maier and D. Brockmann, "Effective containment explains subexponential growth in recent confirmed COVID-19 cases in China," Science, vol. 368, no. 6492, pp. 742-746, 2020, doi: 10.1126/science.abb4557.

[5] Zeynep Ceylan, "Estimation of COVID-19 prevalence in Italy, Spain, and France," Sci. Total Environ., vol. 729, 2020, doi: 10.1016/j.scitotenv.2020.138817.

[6] J. Gnanvi, V. K. Salako, B. Kotanmi, and R. G. Kakaï, "On the reliability of predictions on Covid-19 dynamics: a systematic and critical review of modelling techniques," medRxiv, vol. 6, no. 1, 2021, doi: 10.1101/2020.09.10.20192328.

[7] M. Castro, S. Ares, J. A. Cuesta, and S. Manrubia, "The turning point and end of an expanding epidemic cannot be precisely forecast," Proc. Natl. Acad. Sci., vol. 117, no. 42, pp. 26190-26196, 2020, doi: 10.1073/pnas.2007868117.

[8] D. Mahesh Matta, and M. Kumar Saraf, "Prediction of COVID-19 using machine learning techniques," Bachelor of Science in Computer Science, Blekinge Institute of Technology, Sweden, 2020.

[9] W. C. Roda, M. B. Varughese, D. Han, and M. Y. Li, "Why is it difficult to accurately predict the COVID-19 epidemic?," Infect. Dis. Model., vol. 5, pp. 271-281, 2020, doi: 10.1016/j.idm.2020.03.001.

[10] Calistus N. Ngonghala, Enahoro Iboi, Steffen Eikenberry, Matthew Scotch, Chandini Raina MacIntyre, Matthew H. Bonds, et al., "Mathematical assessment of the impact of non-pharmaceutical interventions on curtailing the 2019 novel Coronavirus," Math. Biosci., vol. 325, 2020, doi: 10.1016/j.mbs.2020.108364.

[11] S. Boccaletti, W. Ditto, G. Mindlin, and A. Atangana, "Modeling and forecasting of epidemic spreading: The case of Covid-19 and beyond," Chaos. Solitons. Fractals, vol. 135, 2020, doi: 10.1016/j.chaos.2020.109794.

[12] P. Melin, J. C. Monica, D. Sanchez, and O. Castillo, "Multiple ensemble neural network models with fuzzy response aggregation for predicting COVID-19 time series: the case of Mexico," Healthcare, vol. 8, no. 2, 2020, doi: 10.3390/healthcare8020181.

[13] T. Sun and Y. Wang, "Modeling COVID-19 epidemic in Heilongjiang province, China," Chaos, Solitons and Fractals, vol. 138, Sep. 2020, doi: 10.1016/j.chaos.2020.109949.

[14] O. Castillo and P. Melin, "A novel method for a covid-19 classification of countries based on an intelligent fuzzy fractal approach," in Healthcare, 2021, vol. 9, no. 2, p. 196.

[15] O. Castillo and P. Melin, "Forecasting of COVID-19 time series for countries in the world based on a hybrid approach combining the fractal dimension and fuzzy logic," Chaos, Solitons \& Fractals, vol. 140, 2020, doi: 10.1016/j.chaos.2020.110242.

[16] N. Donges, "Gradient descent: an introduction to 1 of machine learning's most popular algorithms," 2020.

[17] H. Ritchie, "Coronavirus-source-data." Our World in Data, 2020. [Online]. Available: https://ourworldindata.org/coronavirus-source-data.

[18] Mathworks, "trainlm," MathWork,

2020.

[Online].

Available https://au.mathworks.com/help/deeplearning/ref/trainlm.html.

[19] R. R. O. Al-Nima, M. Al-Kaltakchi, S. Al-Sumaidaee, S. Dlay, W. Woo, T. Han, et al., "Personal verification based on multi-spectral finger texture lighting images," IET Signal Processing, vol. 12, no. 9, 2018, doi: 10.1049/ietspr.2018.5091.

[20] R. R. Al-Nima, S. Dlay, and W. Woo, "A new approach to predicting physical biometrics from behavioural biometrics," International Journal of Computer, Information, Systems and Control Engineering, vol. 8, no. 11, 2014, doi: 10.5281/zenodo.1096924.

[21] R. R. O. Al-Nima, S. S. Dlay, W. L. Woo, and J. A. Chambers, "Efficient finger segmentation robust to hand alignment in imaging with application to human verification," 5th IEEE International Workshop on Biometrics and Forensics (IWBF), 2017, doi: 10.1109/IWBF.2017.7935097.

[22] M. A. M. Abdullah, R. R. Al-Nima, S. S. Dlay, W. L. Woo, and J. A. Chambers, "Cross-spectral iris matching for surveillance applications," Springer, Surveillance in Action Technologies for Civilian, Military and Cyber Surveillance, Chapter 5, 2017, doi: 10.1007/978-3-319-68533-5_5.

[23] R. R. Al-Nima, "Human authentication with earprint for secure telephone system," Iraqi Journal of Computers, Communications, Control and Systems Engineering IJCCCE, vol. 12, no. 2, pp. 47-55, 2012.

[24] M. T. Al-Kaltakchi, R. R. Omar, H. N. Abdullah, T. Han, and J. A. Chambers, "Finger texture verification systems based on multiple spectrum lighting sensors with four fusion levels," Journal of the National Institute of Information and Communications Technology, vol. 1, no. 3, pp. 1-16, 2018, doi: 10.31987/ijict.1.3.28. 
[25] R. R. Al-Nima, "Design a biometric identification system based on the fusion of hand geometry and backhand patterns," Iraqi Journal of Statistical Science, vol. 10, no. 17, pp. 169-180 2010.

[26] Raid Rafi Omar Al-Nima, Moatasem Yaseen Al-Ridha, and Farqad Hamid Abdulraheem, "Regenerating face images from multi-spectral palm images using multiple fusion methods," TELKOMNIKA (Telecommunication, Computing, Electronics and Control), vol. 17, no. 6, pp. 3120-3129, 2019, doi: 10.12928/telkomnika.v17i6.12857.

[27] R. R. O. Al-Nima, N. A. Al-Obaidy, and L. A. Al-Hbeti, "Segmenting finger inner surface for the purpose of human recognition," 2nd International Conference on Engineering Technology and its Applications (IICETA), IEEE, 2019, pp. 105-110, doi: 10.1109/IICETA47481.2019.9012985.

[28] M. R. Khalil, M. S. Majeed, and R. R. Omar, "Personal identification with iris patterns," Raf. J. of Comp. \& Math's., vol. 6, no. 1, 2009, doi: 10.33899/csmj.2009.163762.

[29] Raid R. O. Al-Nima, Tingting Han, Taolue Chen, Satnam Dlay, and Jonathon Chambers, "Finger texture biometric characteristic: a survey," arXiv preprint arXiv:2006.04193, 2020.

[30] Musab T. S. Al-Kaltakchi, Raid Rafi Omar Al-Nima, Mohammed A. M. Abdullah, and Hikmat N. Abdullah, "Thorough evaluation of timit database speaker identification performance under noise with and without the G.712 type handset," Springer, International Journal of Speech Technology, vol. 22, no. 3, pp. 851-863, 2019, doi: 10.1007/s10772-019-09630-9.

[31] R. R. O. Al-Nima, T. Han, and T. Chen, "Road Tracking Using Deep Reinforcement Learning for Self-driving Car Applications", In: R. Burduk, M. Kurzynski and M. Wozniak (eds), Progress in Computer Recognition Systems, CORES 2019, Advances in Intelligent Systems and Computing, vol. 977, Springer, Cham, 2020.

[32] Raid Rafi Omar Al-Nima, Farqad Hamid Abdulraheem, and Moatasem Yaseen Al-Ridha, "Using hand-dorsal images to reproduce face images by applying back propagation and cascade-forward neural networks," $2^{\text {nd }}$ International Conference on Electrical, Communication, Computer, Power and Control Engineering (ICECCPCE19), IEEE, Mosul, Iraq, 13-14 February, 2019, doi: 10.1109/ICECCPCE46549.2019.203755.

[33] Lubab H. Albak, Arwa Hamed, and Raid Rafi Omar Al-Nima, "Design security system based on arduino," TEST Engineering \& Management, The Mattingley Publishing Co., Inc., vol. 82, pp. 3341-3346, January-February 2020.

[34] Moatasem Yaseen Al-Ridha, Raid Rafi Omar Al-Nima, and Ammar Sameer Anaz, "Adaptive neuro-fuzzy inference system for controlling a steam valve," 2019 IEEE 9th International Conference on System Engineering and Technology (ICSET), Shah Alam, Malaysia, 2019, doi: 10.1109/ICSEngT.2019.8906307.

[35] R. R. Al-Nima and S. Kasim, "Picture recognition by using linear associative memory neural network," Tikrit Journal of Pure Science, vol. 13, no. 3, 2008.

\section{BIOGRAPHIES OF AUTHORS}
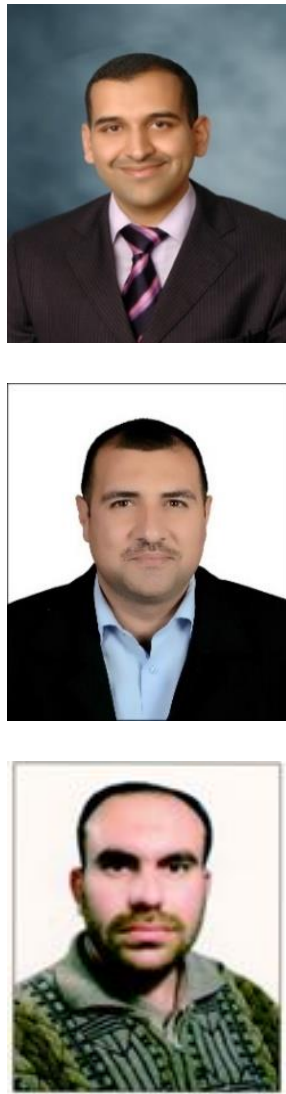

Moatasem Yaseen Al-Ridha received the BSc degrees in Technical Computer Engineering in 2006. In 2013, he accomplished his master's degree in the School of Electrical and Computer Engineering at Southern Illinois University - USA. His research interests are in the fields of Image processing, signal processing, pattern recognition, security, and artificial intelligence.

Ammar Sameer Anaz received the BSc and MSc degrees in Technical Computer Engineering in 2006 and 2018, respectively. During 2018, he worked as an Assistant Lecturer in the Basic Education College in University of Mosul, Iraq. His research interests are in the fields of real time applications, artificial intelligence and image processing.

Raid Rafi Omar Al-Nima received the BSc and MSc degrees in Technical Computer Engineering in 2000 and 2006, respectively. During 2006, he worked as an Assistant Lecturer in the Technical College of Mosul, Iraq. In 2011, he obtained the Lecturer scientific title in the same college. In 2017, he accomplished his $\mathrm{PhD}$ in the School of Electrical and Electronic Engineering at Newcastle University, UK. In 2020, he achieved the title of Assistant Professor in the Northern Technical University. His research interests are in the fields of pattern recognition, security, artificial intelligence and image processing. 